\title{
Systems Thinking for the Enterprise: New and Emerging Perspectives
}

\author{
George Rebovich, Jr. \\ The MITRE Corporation \\ 202 Burlington Road \\ Bedford, MA 01730-1420 \\ grebovic@mitre.org
}

Copyright @ 2006 by The MITRE Corporation. Published and used by IEEE with permission.

\begin{abstract}
New and emerging modes of thought are increasingly being recognized as essential to successful systems engineering in enterprises. This new systems thinking is emerging at the intersection of seminal ideas from modern system thinkers, the broad discipline of information technology, and the theory of complex adaptive systems, particularly those from evolutionary biology and social systems. Part of this new systems thinking requires a replacement of the notion that specific engineering outcomes or goals can always be assured with one that seeks to shape, improve, or increase the value of engineering outcomes through thoughtful interventions in the ever-increasing numbers of circumstances in which we are not fully in control.
\end{abstract}

Keywords: Complexity, systems thinking, evolutionary biology.

\section{Introduction}

Our society and business are in the midst of a major transformation driven by and deriving its character largely from advances in information technology (IT). The rate of technical change in processing, storage, bandwidth, and sensing is enormous. Expansions in other technologies (e.g., biotechnology) have been fueled and shaped by these changes. By dramatically decreasing the cost of information storage and propagation the information revolution is reducing barriers to interactions among people, businesses, organizations, nations, and processes that were previously isolated in space or time. At the same time, future events in this information rich world are harder to predict and control with the result that our world and the systems engineering discipline are becoming increasing complex. Why this is so is illustrated by Figure 1 in which our increasing interconnectedness (left side) makes us all co-producers of outcomes in airline flight availability as we vie for finite resource like non-stop connections whose accessibility and price can change with astonishing speed, as suggested by the on-line flight availability screen shots in the figure.

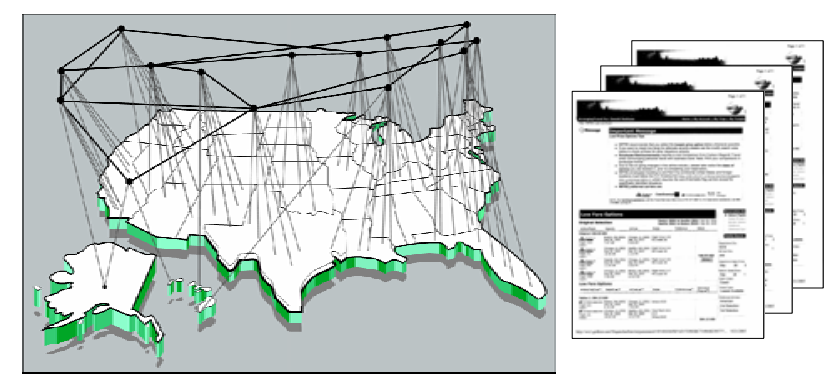

Figure 1. Our World is Becoming Increasingly Complex

In our increasingly connected and complex world we must set our focus on the right level of detail. This requires we differentiate between questions whose answers we can control in a deterministic way from those we are unable to control but whose outcomes we wish to influence or shape. It is the latter to which we must bring our knowledge of "the predictability of general form[s]" to the foreground [1]. The use of general forms to influence or shape systems engineering outcomes is largely the focus of this paper.

Part of our knowledge of general forms comes from the emerging field of complex adaptive systems. Complexity encompasses a number of disciplines but two among them - evolutionary biology and social systems seem particularly relevant to questions and problems of "enterprise as social system" and the role of systems engineer in it. Complexity, with its roots in biology and sociology, together with seminal ideas from modern systems thinkers like Dr. Russell Ackoff and Jamshid Garajedaghi, form the basis for what is called the "new systems thinking" in this paper. 


\section{The Enterprise}

By enterprise we mean an entity comprised of interdependent resources (e.g., people, processes, organizations, technology, funding) that interact with each other (to, e.g., coordinate functions, share information, allocate funding) and their environment to achieve goals [2].

Historically, our focus has been on the technologies which have enabled the development of the piece parts systems and subsystems - contained in the enterprise. Modern systems thinkers like Ackoff and Gharajedaghi are increasingly taking a holistic view of an enterprise as [3].

- a multi-minded, socio-cultural entity,

- comprised of a voluntary association of members who choose their goals and means,

- $\quad$ an entity whose members share values embedded in a (largely common) culture,

- $\quad$ having the attributes of a purposeful entity, and

- $\quad$ an entity whose performance improves through alignment of purposes.

There is a nested nature to many enterprises. At every level, except the very top and bottom, an enterprise itself is part of a larger enterprise and contains sub-enterprises, each with its own people, processes, technologies, funding, and other resources. As notionally depicted in Figure 2, a military Situation Awareness (SA) system is nested (or contained) in an SA constellation which is contained in a Command and Control enterprise.

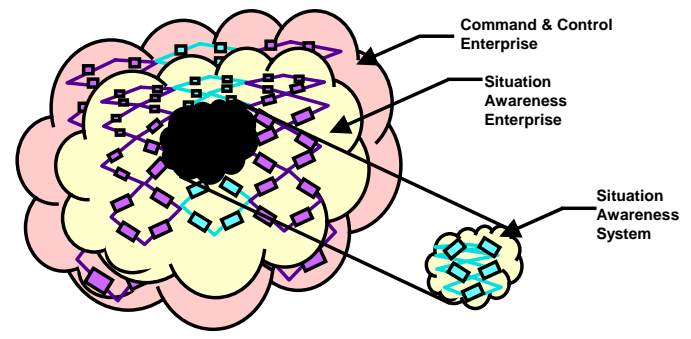

Figure 2. Nested Nature of Hierarchical Enterprises

Alignment of purposes across the levels of the enterprise can improve overall enterprise performance: the sub-enterprise contributes to the goals of the containing enterprise. This view has profound implications for how systems engineers must think about their activities within an enterprise setting: it puts a premium on synthesis [4]. Synthesis is the ability to identify the whole of which a system is a part, explain the behavior or properties of the whole, and disaggregate the whole to identify the role or function of the system in the whole.
At each level in the enterprise hierarchy, the view must be that the current level serves one or more goals of higher levels in the enterprise. This suggests an operational definition of enterprise viewed from the perspective of an individual (system engineer or other participant) or team in the enterprise. The enterprise is the set of interdependent elements (systems and resources) that a participating actor or actors either control or influence. The remainder of the elements constitutes the enterprise environment. This is depicted in Figure 3.

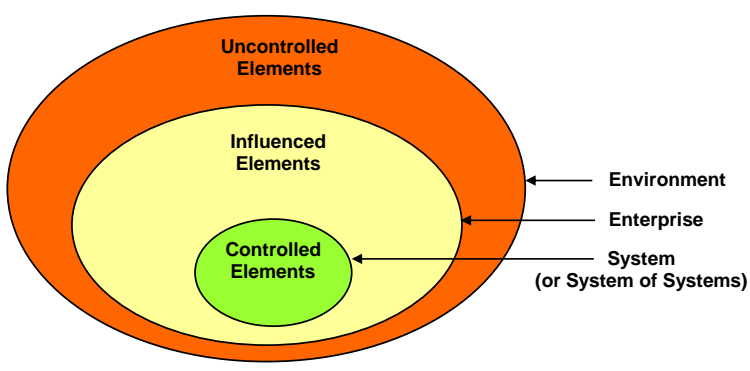

Figure 3. Operational Definition of Enterprise

Note that this definition of enterprise and its boundary are virtual constructs that depend on the make-up, authority, and roles of the participating actors in a community of interest. The implication and view being advocated here is that all actors or teams in an enterprise setting should know "their" enterprise and be aware of which enterprise elements or variables they control and which they influence. Environmental elements or factors cannot be controlled or influenced. But the individual or project team may very well need to be aware of and understand implications of environmental factors.

\section{From Traditional to Enterprise Systems Engineering}

Traditional systems engineering (TSE) is a sequential, iterative development process used to produce products and systems, many of which are of unprecedented technical complication and sophistication. The INCOSE Systems Engineering process, is a widely recognized representation of TSE [5].

An implicit assumption of this TSE process is that all relevant factors are largely under the control of or can be well understood and accounted for by the engineering organization, the system engineer, or the program manager and this is normally reflected in a TSE culture.

There are fundamental differences in an enterprise. While some factors may continue to be well understood by or remain under the control of the system engineer or program manager, others are not. Because enterprises embrace diverse agencies, sponsors, and operational communities, there is increased emphasis on working across and bridging organizational cultures, agendas, and 
socio-political-economic differences. Enterprises exhibit attributes of a complex system. As a result there arise questions of how to deal with enterprise processes that approximate natural evolution and, in some cases, how to deliberately mimic, encourage, facilitate, and channel them in constructive directions. Adjectives that are used to describe system engineering and other processes in an enterprise expand to include: evolutionary, emergent, adaptive, self-organizing, competitive, and cooperative. A generalization of the INCOSE Systems Engineering process may be more appropriate in an enterprise environment [6].

\section{A Framework for Harnessing Complexity}

A consequence of the evolution to an enterprise model is that each of us, as participating actors, are losing the ability to control many enterprise elements while at the same time we are gaining in our potential to influence many others (ref. Figure 3). Systems engineering, therefore, is becoming more and more about the ability to influence in our increasingly complex environment [7].

\section{A General Development Framework for Complex Enterprises}

Development is a purposeful transformation towards higher levels of differentiation and integration at the same time. Development is an evolutionary process by which a social system (e.g., individual, team, community, enterprise) increases its ability or value (as gauged by performance, effectiveness, impact, influence, profitability, etc.). Differentiation refers to deviations among entities that are apparently similar and integration refers to the similarities among things that are apparently different. The former emphasizes tendencies toward increased complexity, variety, and autonomy while the latter tends toward increased order, uniformity, conformity, and collectivity. The seemingly opposite tendencies of differentiation and integration complement each other to create innovation which is a journey or process as depicted in Figure 4.

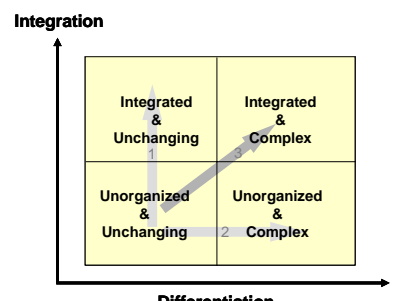

Differentiation

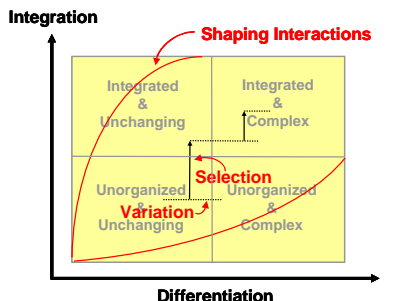

Differentiation
Figure 4. SE Process Moving to ESE

Referring to the left side of Figure 4, an enterprise in the lower left quadrant is static and unorganized. Change is infrequent or non-existent and the piece-parts that are there are unorganized into any semblance of a coherent whole. Movement or evolution is generally possible, of course, so the question becomes one of direction.

One evolutionary path is to organize all the elements and processes that currently exist in the enterprise, that is, integrate straight up to the upper left quadrant. The result is organized simplicity: a highly integrated organization which knows how to do things very well in essentially one way. Change in the way things are done is difficult to effect. The organization is rigid and inflexible in how it responds to problems, opportunities, and other changes in its environment.

Another possibility for evolution is towards unorganized complexity. This is an organization that proliferates many ideas for accomplishing activities or developing products (differentiation) but never integrates them into a coherent process or product. Frequently, there is an element of imagination in this differentiation but what keeps it from being innovation is that the good ideas never get realized. Examples of unorganized complexity are some of the early internet inventors who produced numerous elegant and novel approaches to solving internet technology problems but never put them together into a coherent, financially profitable business package.

The last possibility for evolution is towards integrated complexity. This is a movement towards complexity and order at the same time. This is an organization that proliferates many new ideas about its processes, products, and solution approaches and then selects and integrates the best of them into subsequent versions or entirely new incarnations. Some organizations develop elaborate and detailed 10-year plans and then execute to that plan. An integrated, complex organization is not like that: instead, it "reinvents itself" through continual differentiation and integration to higher levels of value and performance. This "continuous reinvention" provides adaptability to environmental changes.

Complex entities do not develop in straight lines, nor are specific outcomes necessarily guaranteed or predictable; however, their development can be controlled through a guiding developmental framework based on variation of technical strategies, interaction of the enterprise's building and governing agents, and selection of winning strategies through market-like mechanisms. These are interlocking concepts that can generate productive actions in complex situations that cannot be controlled but can be influenced. The framework helps ask and answer the question, "what interventions in the enterprise are likely to bring us to a future we would prefer?" This moves us from being passive observers in complex situations to active participants who manage variation, shape interactions, and make selections to guide, even accelerate improvement over time. A variation-interaction-selection framework clusters the 
discussion of change mechanisms in a complex system on three central and connected questions [8]:

- What is the right balance between variety and uniformity in the enterprise strategies and standards?

- What (or who) should interact with what (or whom) and when?

- Which strategies and standards should be adopted and what should be eliminated?

\section{Variation: The Source of Innovation and Adaptation}

Variation produces raw material for adaptation. The key question in variation surrounds choosing the right balance between variety and uniformity. There can be substantial benefits to individual users that derive from a large number of other users of a single, common standard. When a lead type becomes the de facto "best" and environmental conditions are not changing, rapid convergence on a standard can result in large economy of scale benefits to both the user and the industry producing it. But in changing environmental conditions or when types available so far are not the best possible, loss of variety (premature convergence) can result in potentially superior alternatives never being considered. Within the enterprise there is the question of whether and when to converge on a strategy or technical standard and these same forces are at work.

The actions available to shape the behavior of a complex system often work not just by accommodating variety; they also work by actually increasing or decreasing the variety of agents in a population (e.g., internet standards, product designs under consideration for development). Variety turns up repeatedly in complex systems as a crucial factor in their development. But the situation is not always as simple as saying that homogeneity is bad and variety is good. Homogeneity can be very useful indeed, as the following example shows.

Example: Consider a C2 Enterprise built on a layered architecture in which IP is a chosen point of convergence for all network implementations [9]. The standardization of IP is a form of homogeneity imposed on the enterprise. Differentiation is lost at that particular level of the architecture and innovation is - if not lost - seriously affected because the inertia of a widely accepted and adopted standard can only be overcome by the most compelling reasons. But that same standardization enables innovation to flourish above and below the standard and is therefore a net gain for the enterprise.

So, the key question in variation surrounds choosing the right balance between variety and uniformity.
Exploration versus exploitation is an important tradeoff between the creation of untested strategies and standards that may be superior to that which currently exists as compared to the adoption of tested standards that have so far proven best. This trade-off is important across a wide range of situations in which the testing of new or emerging standards comes at some expense to realizing benefits of those already available.

There are no hard and fast rules for when one would reliably do better with more variety. In an "ideal situation" where the current approach to a problem is judged the "best possible" and the problem or environment is unlikely to change, exploration is not indicated. For other situations there are some broad conditions in which exploration is likely to be of value. These are summarized in the table below, along with Rules of Thumb.

Table 1. Rules of Thumb on Encouraging Variety

\begin{tabular}{|l|l|}
\hline \multicolumn{1}{|c|}{ Condition } & \multicolumn{1}{c|}{ Rules of Thumb } \\
\hline $\begin{array}{l}\text { When problems are long- } \\
\text { term or widespread. }\end{array}$ & $\begin{array}{l}\text { - The more use that can be made of } \\
\text { an improvement, the more it pays } \\
\text { to bear the costs of searching for } \\
\text { one. }\end{array}$ \\
\hline $\begin{array}{l}\text { Problems that provide fast, } \\
\text { reliable feedback. }\end{array}$ & $\begin{array}{l}\text { - If you can learn quickly and } \\
\text { reliably whether an alternative } \\
\text { solution might be better then you } \\
\text { have more chances to find an } \\
\text { improvement, and you have longer } \\
\text { to gain from what you might } \\
\text { discover. }\end{array}$ \\
$\begin{array}{ll}\text { - Where fast and accurate feedback } \\
\text { channels don't exist, try to create } \\
\text { them so the benefits of exploration } \\
\text { can be gained. }\end{array}$ \\
\hline $\begin{array}{l}\text { Problems with low risk of } \\
\text { catastrophe from } \\
\text { exploration. }\end{array}$ & $\begin{array}{l}\text { If the risk of an extremely bad from exploration can be } \\
\text { judged as low then consider } \\
\text { increasing the amount you are } \\
\text { willing to do. } \\
\text { - Try to create ways to lower risk of } \\
\text { extremely bad results so the } \\
\text { benefits of exploration can be } \\
\text { gained. }\end{array}$ \\
\hline $\begin{array}{l}\text { Problems that have looming } \\
\text { disasters. }\end{array}$ & $\begin{array}{l}\text { If continuing to exploit the best } \\
\text { solution found to date will likely to } \\
\text { lead to disaster, then explore. } \\
\text { - The relative attractiveness of } \\
\text { exploring comes from the negative } \\
\text { yield of exploiting. }\end{array}$ \\
\hline
\end{tabular}

\section{Interaction: The Shaping of the Enterprise}

Interaction makes or changes the rules agents play by and their interaction patterns. Interaction among agents shapes the creation and destruction of variety and produces the events that drive selection. When thinking about the variety of agents and their strategies, one is led to the question of the right balance between variety and uniformity. This balance is achieved partially through 
interaction which seeks to answer, "what or (who) should interact with what (or who) and when?"

More fundamentally, and beyond manipulating variety and uniformity, interactions help shape the outcome space within which a complex system develops as depicted in the right side of the figure above. Events of interest within an enterprise arise (or do not) from the interactions of its agents with each other and with the enterprises' strategies and standards.

Interaction patterns shape the events in which members of an enterprise become directly involved and they provide the opportunity for spreading and recombining of strategies and standards that lead to their creation and destruction. The events drive processes of selection and amplification that ultimately change the frequency and variety of strategies and standards. Interaction patterns help determine which strategies and standards will be successful for the agents and the enterprise and this, in turn, will help shape the dynamics of the interaction patterns themselves.

\section{Selection: The Integration and Exploitation of Value}

Selection promotes adaptation. The process of selection promotes adaptation by amplifying success at the level of the product or of strategy or standard. A product may be thought of as a collection of standards. This involves making decisions on which products, standards or strategies should be proliferated and which eliminated. Selection at the two levels can work in very different ways. Product selection duplicates an entirely new product without the need to determine or know precisely the cause of success. Strategy selection picks a specific standard from among a number used by many products. This requires an explicit decision about what standard for the products' success.

\section{An Example: Military Net Centricity}

Consider an enterprise consisting of organizations that develop, field and operate its systems. To use the military C2 enterprise as an example, it would include government organizations and commercial companies that form the acquisition community, as well as military operational organizations that employ the systems in the execution of their roles and responsibilities.

Systems of record (SoRs) are developed by the acquisition community through funded programs of record (PoRs) using TSE methods and processes. The PoRs create a plan to develop a system capability and execute the plan. This is notionally depicted in figure 5 by the horizontal lines that illustrate the development of SoRs through TSE using program funds. The TSE process works well when the system requirements are relatively well known, technologies are mature, and the capabilities to be developed are those of the system, per se, and not of the enterprise.

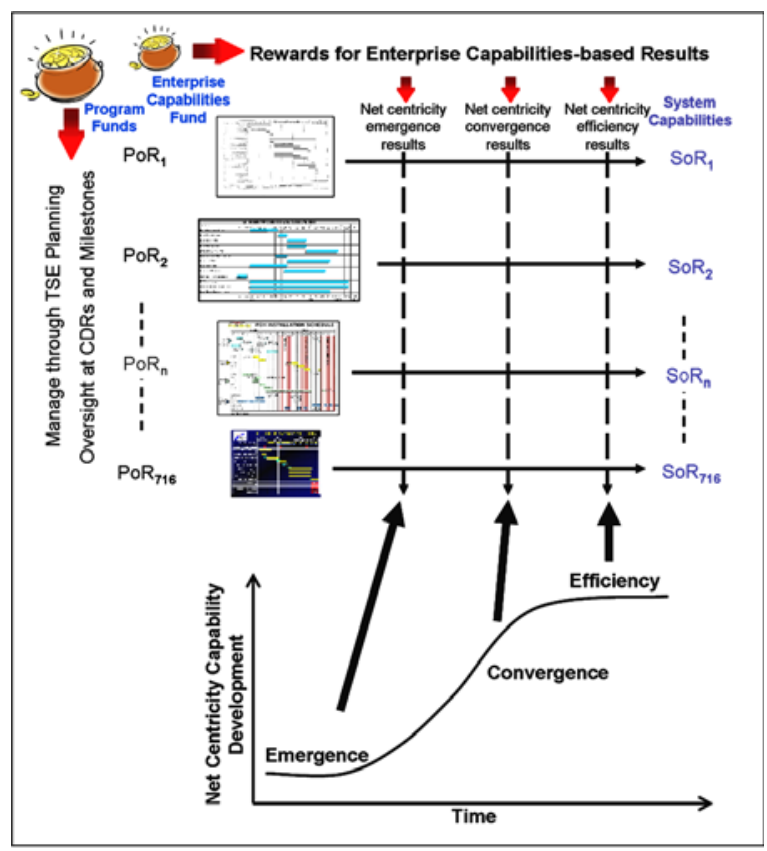

Figure 5. Development of System Capabilities and Evolution of Enterprise Capabilities

Enterprise capabilities, by their very nature, involve contributions from multiple components of the enterprise (e.g., interoperability). Some enterprise capabilities are not knowable in advance of their appearance (e.g., net centricity, jointness). This occurs for many reasons: e.g., needed technologies and standards are still emerging and it is not yet known which will achieve market dominance, and the realization of operational concepts for the enterprise capability involves the participation of many SoRs and of the operational units that employ them. Enterprise capabilities evolve like the s-curve depicted in the lower part of figure 5 and so require a different approach from TSE.

Figure 5 illustrates an approach that shapes the evolution of enterprise capabilities through a supplier model (employing results-based rewards) at the same time that individual system capabilities are being developed via the TSE approach of contracting for a promise of the future delivery of a system capability.

This involves the creation of a separate enterprise capabilities fund (which, of course, may be derived from the program funds) and management different from the PoRs. The basic notion is to stimulate innovation by and interactions among PoRs by rewarding ones that move the enterprise towards net centricity. Reward criteria depend on the phase of net centricity the enterprise is in. In the emergence phase, reward criteria would favor variety and exploration of standards, strategies and solution 
approaches; in convergence the reward criteria would balance exploration and exploitation and in the efficiency phase, the criteria would reward uniformity, integration and exploitation.

Net centricity is a characteristic of the military C2 enterprise primarily in its operation. In addition, there are strong elements of social structure and dynamics in the evolution of net centric capabilities as well as its utilization in net centric operations (NCO). As a result, the characterization of the military $\mathrm{C} 2$ enterprise $\mathrm{NCO}$ performance should be strongly tied to the behavior of operational units employing SoRs in actual operations and involve sociologists as well as operational experts and technologists. Examples of criteria in the emergence phase might include: an increase in the total number of interface control documents (ICDs) among all PoRs and an increase in the volume of voice, email, chat and instant messaging among operational platforms. Criteria in the convergence phase might include: a decrease in the number of ICDs among PoRs and less episodic, more continuous interactions among operational platforms.

\section{Summary}

Enterprises are complex systems. Enterprise systems engineers are losing the ability to control many enterprise elements at the same time we are gaining the potential to influence others. Systems engineering is becoming more about the ability to influence in an increasingly complex environment. This paper has presented a framework to help answer, "what interventions in the enterprise are likely to bring us to a future we would prefer?" This makes us active participants who manage variation, shape interactions, and make selections to guide and accelerate enterprise improvements over time.

\section{Acknowledgements}

The author acknowledges Dr. Joseph K. DeRosa, Mr. Michael L. Kuras, and Dr. Robert S. Swarz of The MITRE Corporation, who contributed to the development of a number of the ideas presented herein.

\section{Biography}

George Rebovich leads the Aerospace Situation Awareness Systems section for the MITRE Corporation's Center for Air Force C2 Systems. He holds a B.S. degree (Mathematics) from the U.S. Military Academy, an M.S. degree (Mathematics) from Rensselaer Polytechnic Institute, a certificate of Administration and Management from Harvard University and is a graduate of the U.S. Army Command and General Staff College. He has held various systems engineering positions at MITRE. He served in the U.S. Army before joining MITRE including tours of duty in the United States, Europe, and Southeast
Asia. He is a former Assistant Professor of Mathematics at the U.S. Military Academy.

\section{Disclaimer}

The author's affiliation with The MITRE Corporation is provided for identification purposes only, and is not intended to convey or imply MITRE's concurrence with, or support for, the positions, opinions or viewpoints expressed by the author.

\section{References}

[1] Gould, S. J., 1989, Wonderful Life, New York, NY: W. W. Norton \& Company, p. 290.

[2] 17 December 2004, "Enterprise in the Net Centric Implementation Framework,” V1.0.0, NESI.

[3] Gharajedaghi, J., 1999, Systems Thinking: Managing Chaos and Complexity, Boston, MA: Butterworth Heinemann.

[4] Ackoff, R., 15 February 2005, Systems Thinking and its Radical Implications for Management, IMS Lecture/Boston.

[5] June 2004, “Systems Engineering Handbook," INCOSE-TP-2003-016-02, Version 2a, INCOSE.

[6] DeRosa, J. K., G. Rebovich, Jr., and R. S. Swarz, 2006, “An Enterprise Systems Engineering Model," Proceedings of INCOSE 2006 International Symposium. To be published.

[7] Rebovich, G., Jr., November 2005, Enterprise Systems Engineering Theory and Practice, Volume 2: Systems Thinking for the Enterprise: New and Emerging Perspectives, MP05B043 Vol 2, The MITRE Corporation, Bedford, MA.

[8] Axelrod, R. and M. D. Cohen, 2000, Harnessing Complexity: Organizational Implications of a Scientific Frontier, Basic Books.

[9] USAF Electronic Systems Center Strategic Technical Plan (STP) V 2.1. 